\title{
Demand-Driven Scheduling of Movies in a Multiplex
}

\author{
Jehoshua Eliashberg, Quintus Hegie, Jason Ho, Dennis Huisman, \\ Steven J. Miller, Sanjeev Swami, Charles B. Weinberg \\ and Berend Wierenga
}

\begin{tabular}{|l|l|}
\hline \multicolumn{2}{|l|}{ ERIM REPORT SERIES RESEARCH IN MANAGEMENT } \\
\hline ERIM Report Series reference number & ERS-2007-033-MKT \\
\hline Publication & May 2007 \\
\hline Number of pages & 33 \\
\hline Persistent paper URL & http://hdl.handle.net/1765/10069 \\
\hline Email address corresponding author & bwierenga@rsm.nl \\
\hline Address & Erasmus Research Institute of Management (ERIM) \\
& RSM Erasmus University / Erasmus School of Economics \\
& Erasmus Universiteit Rotterdam \\
& P.O.Box 1738 \\
& 3000 DR Rotterdam, The Netherlands \\
& Phone: $\quad+31104081182$ \\
& Fax: $\quad+31104089640$ \\
& Email: info@erim.eur.nl \\
& Internet: $\quad$ www.erim.eur.nl \\
\hline
\end{tabular}

Bibliographic data and classifications of all the ERIM reports are also available on the ERIM website: www.erim.eur.nl 


\section{ERASMUS RESEARCH INSTITUTE OF MANAGEMENT}

\section{REPORT SERIES}

\section{RESEARCH IN MANAGEMENT}

\begin{tabular}{|l|l|}
\hline ABSTRACT AND KEYWORDS \\
\hline Abstract & $\begin{array}{l}\text { This paper describes a model that generates weekly movie schedules in a multiplex movie } \\
\text { theater. A movie schedule specifies within each day of the week, on which screen(s) different } \\
\text { movies will be played, and at which time(s). The model consists of two parts: (i) conditional } \\
\text { forecasts of the number of visitors per show for any possible starting time; and (ii) an } \\
\text { optimization procedure that quickly finds an almost optimal schedule (which can be } \\
\text { demonstrated to be close to the optimal schedule). To generate this schedule we formulate the } \\
\text { so-called movie scheduling problem as a generalized set partitioning problem. The latter is } \\
\text { solved with an algorithm based on column generation techniques. We have applied this } \\
\text { combined demand forecasting Ischedule optimization procedure to a multiplex in Amsterdam } \\
\text { where we supported the scheduling of fourteen movie weeks. The proposed model not only } \\
\text { makes movie scheduling easier and less time consuming, but also generates schedules that } \\
\text { would attract more visitors than the current 'intuition-based' schedules. }\end{array}$ \\
\hline Free Keywords & Optimization of movie schedules, Integer programming, Column generation, Demand forecasting \\
\hline Availability & $\begin{array}{l}\text { The ERIM Report Series is distributed through the following platforms: } \\
\text { Academic Repository at Erasmus University (DEAR), DEAR ERIM Series Portal } \\
\text { Social Science Research Network (SSRN), SSRN ERIM Series Webpage } \\
\text { Research Papers in Economics (REPEC), REPEC ERIM Series Webpage }\end{array}$ \\
\hline Classifications & $\begin{array}{l}\text { The electronic versions of the papers in the ERIM report Series contain bibliographic metadata } \\
\text { by the following classification systems: } \\
\text { Library of Congress Classification, (LCC) LCC Webpage } \\
\text { Journal of Economic Literature, (JEL), JEL Webpage } \\
\text { ACM Computing Classification System CCS Webpage } \\
\text { Inspec Classification scheme (ICS), ICS Webpage }\end{array}$ \\
\hline
\end{tabular}




\title{
Demand-Driven Scheduling of Movies in a Multiplex
}

\author{
Jehoshua Eliashberg \\ The Wharton School, University of Pennsylvania, Philadelphia, Pennsylvania 19104 \\ eliashberg@wharton.upenn.edu \\ Quintus Hegie \\ School of Economics, Erasmus University Rotterdam, The Netherlands, \\ quintus@quintushegie.com \\ Jason Ho \\ School of Business Management, Ryerson University, Toronto, ON, M5B 2K3 Canada, \\ jasonho@ryerson.ca \\ Dennis Huisman
}

Econometric Institute, Erasmus University Rotterdam, The Netherlands, huisman@few.eur.nl

Steven J. Miller

Mathematics Department, Brown University, Providence RI 02912,sjmiller@math.brown.edu

Sanjeev Swami

Department of Management, Faculty of Social Sciences, Dayalbagh Educational Institute,

Agra - 282005, UP, INDIA, sswami1853@gmail.com

Charles B. Weinberg

Sauder School of Business, University of British Columbia, Vancouver, BC V6T 1 Z2 Canada weinberg@sauder.ubc.ca

Berend Wierenga

RSM Erasmus University Rotterdam, The Netherlands, bwierenga@rsm.nl

May 1, 2007

NOT FOR REPRODUCTION OR QUOTATION WITHOUT WRITTEN PERMISSION OF THE AUTHORS

\begin{abstract}
This paper describes a model that generates weekly movie schedules in a multiplex movie theater. A movie schedule specifies within each day of the week, on which screen(s) different movies will be played, and at which time(s). The model consists of two parts: (i) conditional forecasts of the number of visitors per show for any possible starting time; and (ii) an optimization procedure that quickly finds an almost optimal schedule (which can be demonstrated to be close to the optimal schedule). To generate this schedule we formulate the so-called movie scheduling problem as a generalized set partitioning problem. The latter is solved with an algorithm based on column generation techniques. We have applied this combined demand forecasting /schedule optimization procedure to a multiplex in Amsterdam where we supported the scheduling of fourteen movie weeks. The proposed model not
\end{abstract}


only makes movie scheduling easier and less time consuming, but also generates schedules that would attract more visitors than the current 'intuition-based' schedules.

Key words: optimization of movie schedules, integer programming, column generation, demand forecasting.

\section{Introduction}

The motion picture industry is a prominent economic activity with total world wide box office revenue of $\$ 25.82$ billion in 2006, of which $\$ 9.49$ billion is in the U.S.A ${ }^{1}$. Movie forecasting and programming in practice tend to be associated with intuition rather than analysis and this also characterizes the tradition of decision making in this domain. However, many problems in the film industry are actually quite amenable to model building and optimization, and this is increasingly recognized by movie executives. In this paper we focus on one such problem: the detailed scheduling of a movie theater.

The movie industry runs on a weekly cycle. A movie program or schedule in a theater is designed for a week, and in the U.S.,for example, each Friday a new movie week starts. Therefore, a movie theater has to prepare a new movie program every week. This is particularly complex for multiplex theaters, the increasingly dominant movie theater format around the world. For example in the Netherlands, which is the setting of the empirical part of this paper, multiplexes with 8 or more screens represent $24 \%$ of all the movie theater seats, and in $2005,34 \%$ of total box office. It is clear that the programming of such large cinemas is not an easy matter.

For each week's movie program, management must determine what movies will be shown, where they will be shown (on which screens), on which days, and at what times during the day. Typically, on each screen, a theater can accommodate 3 to 5 showings per days, where a "showing" is defined as the screening of one movie, including trailers and advertisements. This means that a 10 -screen theater needs to program around 280 showings per week. At present, this programming is mostly done manually with pencil and paper, by specialists in the theater company. These programmers combine an analytical mind with a broad knowledge about movies and about the audience of individual theaters, often based on many years of experience. It is our belief that an analytical system can help here. This would not only relieve

\footnotetext{
${ }^{1}$ MPAA Annual Report 2006
} 
theaters from a labor-intensive task recurring every week, but we believe that an analytically based system will perform even better than the current manual procedure.

The programming problem for an individual movie theater consists of two stages: (i) the selection of the list of movies, i.e., the movies to be shown in the particular week and (ii) the scheduling of these movies over screens, days, and times of the day. Stage (i) includes making agreements with movie distributors and is completed before stage (ii). In this study we develop a solution for the second stage, which involves constructing detailed schedules for where (which screen(s)) and when (days, times) the different movies will play. For analytical procedures that deal with the first stage, i.e., deciding on the movies to be shown in a particular week, see Swami, Eliashberg and Weinberg (1999), and Eliashberg, Swami, Weinberg and Wierenga (2001).

Our scheduling problem has two sub-problems. First, we need an answer to the following question: if a particular movie would be shown on a particular day, at a particular time, how many visitors would it attract? Inputs for making these forecasts are observed visitors numbers in the previous weeks (for existing movies), characteristics of the movie (for newly released movies), and information about variables such as specific events (holidays) and the weather. Making such conditional forecasts is not an easy task and it belongs to the realm of the marketing discipline. Second, given this demand assessment, we have to find the schedule that maximizes the number of visitors for the week, given constraints such as theater capacity and runtimes of the movies. This is a non-trivial problem, for which we need the discipline of Operations Research. The specific solution approach employed here is column generation, a method designed to solve (integer) linear optimization problems with many variables/columns, but relatively few constraints. The remainder of this paper describes how by combining the two disciplinary approaches a solution to the movie scheduling problem was obtained.

The paper first takes a closer look at the movie scheduling problem (Section 2). In Section 3 we describe the column generation approach to the optimization problem. In Section 4, we discuss the method that we developed to conditionally forecast the number of visitors of a show. Then, in Section 5, we 
apply the complete procedure, through a model we call SilverScheduler, to fourteen movie weeks of the theater De Munt, a multiplex with 13 screening rooms in central Amsterdam. With over 1 million visitors a year, this is the second largest theater in the Netherlands. The last part of the paper (Section 6) discusses the results, puts them in perspective and discusses issues for future research.

\section{Problem Description and the Research Project}

As movie theaters have evolved from single screen theaters to multiplexes and even megaplexes, the problems of scheduling movies onto screens has become increasingly complex. Some of the key reasons for that complexity are as follows.

First, there are a large number of different movies that the theater wants to show in a typical week. This number is typically larger than the number of screens. Moreover, these movies have different running times. For example, over the different movies running in the De Munt theater in Amsterdam during our observation period, the running time was as short as 71 minutes for Plop en Kwispel (a kids movie) and as long as 240 minutes for Ring Marathon.

Second, the number of seats per screening room differs. In the De Munt theater the smallest room has 90 seats and the largest room, 382. For some movies there are contractual arrangements with distributors about the specific screening room in which a movie will be shown. This is typically the case for newly released movies, which distributors like to have in large rooms. For other movies, the theater management is completely free where to screen them.

Third, there is variability in demand for movies. In our sample, for example, in May 2005, the most popular movie drew 8027 visitors in a week and the least popular 133 visitors. In addition, movie demand varies by time of day and day of week. For example, we found that Saturday evening at $8 \mathrm{pm}$ is the most appealing time for movie goers. The expected demand for a movie influences what screening room it will be shown in; the demand for some movies may be so large that it could be double or triple booked. 
Fourth, there are different genres of movies, and this also has implications for their scheduling. For example, children's movies should preferably be shown at times when children are free from school (weekend and Wednesday afternoon ${ }^{2}$ ), and will not be shown during the evenings.

Fifth, there are many constraints posed by the logistics of a movie theater. An obvious limitation is the time that the theater is open. (The closing time is often determined in part by the public transportation schedule: non-drivers attending the last show need to be able to get home). Other logistical constraints are the time needed for cleaning the room after a showing (dependent on the size of the room), and the capacity limitations of the ticket office, the corridors, the staircases, and the concession sales counters. To provide high consumer satisfaction with the theatrical experience, crowding should be avoided as much as possible, and major movies with many visitors should not start at the same time, especially if they are on the same floor.

Finally, management may impose specific constraints which are expected to contribute to the revenue of the theater. For example, for the theater in our study, management specified that the time between two movie starts should not be more than 20 minutes. In that case an impulse movie visitor, looking for entertainment without strong preference for a particular movie, never has to wait more than 20 minutes.

We take the perspective of management for this problem, which is to maximize the expected number of visitors to that theater for a given week. Another possible objective is profit maximization. However, the cost and revenue structure are such that at this level, attendance maximization and profit maximization are likely to provide quite similar results. We confirmed this with empirical testing.

Consequently, the problem to be solved is how to generate a schedule of the different movies on the different screens during a given week, which obeys all the requisite constraints and maximizes the number of visitors.

\section{The Study}

Most theaters solve this scheduling problem by hand. "Movie programmers" work with hardcopy planning sheets to fill the total capacity of the theater, taking into account the various constraints to

\footnotetext{
${ }^{2}$ Primary school children in the Netherlands have free time on W-ednesday afternoons.
} 
the extent possible. In doing so, programmers follow certain procedures (e.g., choose the size of the screening room for a movie, dependent on the expected attendance), and use a mixture of hard facts (e.g., attendance figures of the past week) and intuition (e.g., the effect of an important soccer match on TV on the number of theater visitors for a movie). It takes a lot of experience to become a skilled movie programmer. And even then the solution is never perfect. For a human mind it is practically impossible to find the best possible solution while at the same time dealing with all the constraints. Moreover, the schedules have to be made under time pressure. As mentioned, in a movie theater every Thursday/Friday ${ }^{3}$ a new movie program starts running, which has to be finalized on the preceding Monday. The critical time window is the Monday morning after the attendance figures of the recent weekend have become available and before the information about the new schedule has to be sent to the newspapers and posted online.

The purpose of this study is to develop a procedure that makes it possible to automate the movie programming process as described above. We developed a mathematical procedure that produces the (almost) optimal schedule, given the demand information and the various constraints. Such an algorithm should work fast and should deliver the output in a format that can directly be entered in the theater's planning procedure. It should also be easy for a manager to make last-minute changes to the schedule as recommended by the procedure. In decision situations like this, there can always be new information, (e.g., about a strike, a sudden change in the weather), that is not included in the model and that may induce the manager to make last minute changes in the schedule. The generation of a recommended schedule can be automated, but for the implementation of a schedule, human judgment still remains vital.

We were asked to work on this problem by Pathé, the largest movie exhibitor in the Netherlands. They view the current manual programming procedure as being too cumbersome and time-consuming. Our purpose was not to just save the time of the programmer, but also to do a better job in terms of generating movie schedules that may attract larger numbers of visitors. It was decided to take the setting of one particular Pathé location, the De Munt Theater, as our empirical environment. Management provided complete access to the internal data they had, and closely monitored the project and its results.

\footnotetext{
${ }^{3}$ In the Netherlands the new movie week starts on Thursday, in the USA and Canada this is on Friday.
} 
A key input to the scheduling algorithm is the demand information about the movies in the movie list. For each movie in the movie list we need to forecast the number of visitors that this movie will generate in any given showing. This estimate has to be available for each day of the week and for each different possible starting time of the movie. For this purpose, we developed a forecasting procedure with two modules. The first module is for movies that have been running already. The observed numbers of visitors are used to estimate a forecasting model. The second module is for newly released movies, where the numbers of visitors are forecasted in an indirect way, using the characteristics of a movie as predictor variables. These forecasting procedures are described after the discussion of the scheduling algorithm.

We call the complete model, the scheduling algorithm integrated with the demand assessment procedure, SilverScheduler. After the description of the scheduling part and the forecasting part of SilverScheduler, we discuss its implementation at the theater De Munt.

\section{A Column Generation Approach to Solve the Movie Scheduling Problem}

To produce a movie program for a certain week, we need to find schedules for the different days in that week. We define the movie scheduling problem (MSP) as the problem of finding the optimal movie program for a single day given the list of movies to be shown (the "movie list"), the running times of these movies, the demand figures for these movies, the capacities of the different screening rooms, information about contractual agreements with distributors about screening rooms for particular movies, and which takes into account the different constraints as mentioned in Section 2. In Section 5, we describe a procedure to come up with a week schedule by solving a sequence of MSPs.

The MSP has many similarities with other well-known scheduling problems such as vehicle scheduling/routing, aircraft scheduling/routing, and crew scheduling (or combinations of these problems). These kinds of problems are often formulated as set partitioning or covering problems (eventually with additional constraints). We also use a set partitioning type of formulation. A drawback of such a formulation is the large number of variables, which can be overcome by using column generation techniques. We refer to Barnhart et al. (1998), Lübbecke and Desrosiers (2005), and Desaulniers et al. (2005) for a gener- 
al introduction on column generation. Some recent references to applications of column generation to the aforementioned scheduling problems are Löbel (1998) for vehicle scheduling, Desaulniers et al. (1997) for aircraft routing and scheduling, Huisman et al. (2003) for integrated vehicle and crew scheduling, and Sandhu and Klabjan (2006) for integrated airline plan.

\subsection{Mathematical Formulation}

Before we present the mathematical formulation of the MSP, we first introduce some notation. Analogous to the so-called time-space networks in transportation problems such as vehicle and crew scheduling, we use here time-movie networks. These networks are acyclic directed graphs denoted by $G^{s}=\left(N^{s}, A^{s}\right)$, and are defined for each screen $s$. Furthermore, let $S$ and $M$ be the set of screens and movies, respectively. Recall that due to capacity restrictions and contractual agreements not all movies can be shown on each screen, therefore we define $M^{s}$ as the subset of movies that can be shown on screen $s$. We denote for each movie $m$ its duration and cleaning time as $d r_{m}$ and $c l_{m}$, respectively. Define $T$ as the set of possible time points at which a movie can start, where $t_{i}$ is the time corresponding to time point $i$. In each graph, a node $(i, m)$ corresponds to starting a movie $m$ on time point $i$ on screen $s$. Moreover, a source and a sink are defined. There are arcs from the source to all intermediate nodes, and from them to the sink. If on a screen only one movie is allowed to play throughout the day, an arc is defined between each pair of nodes $(i, m)$ and $(j, n)$ if $t_{j} \geq t_{i}+d r_{m}+c l_{m}$ with $m=n$. In Figure 1, the time-movie network is depicted for this particular case. For instance, there is an arc between nodes $(1,1)$ and $(3,1)$, because the duration and cleaning time of movie 1 is not larger than the time between time points 1 and 3. A path in the network corresponds to a feasible schedule for the whole day on one screen. In this case, the dotted path corresponds to showing movie 1 on time points 1 and 3. 


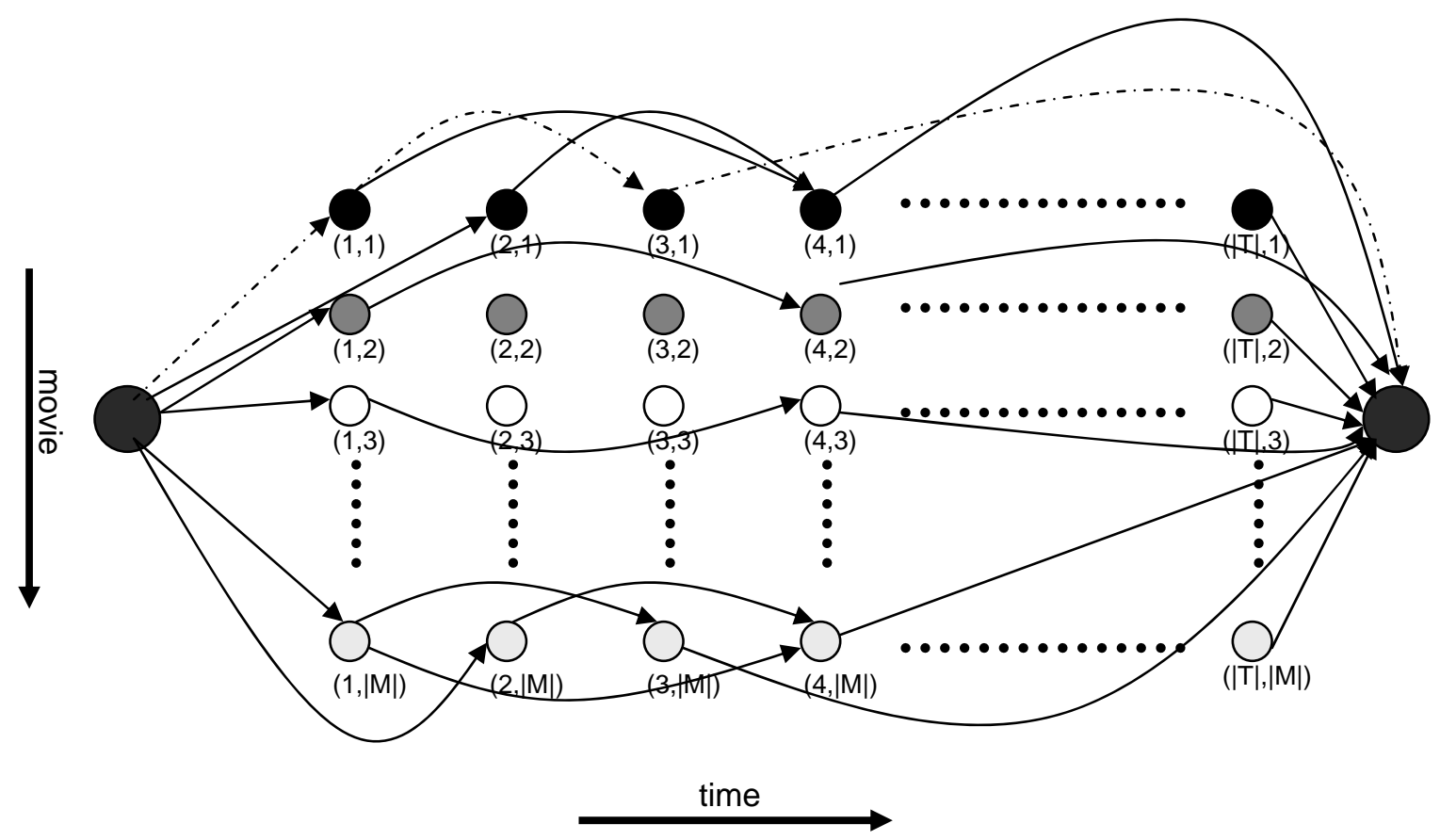

Figure 1: A time-movie network for one screen (with one movie per screen)

If two movies can be shown on the same screen, the network is extended to two layers. In each individual layer, there are only arcs between nodes corresponding to the same movie, while the arcs between the layers are between nodes with different movies. Since a switch of movie on a screen is not preferred, a penalty $Q$ is introduced when the arc between the different layers is chosen. In these networks each path from source to sink is a feasible schedule for one screen. The cost of a path is defined as $c_{p}$, which is equal to the sum of the costs of the individual arcs in the path. Each arc $(i, m, j, n)$ has as costs -min $\left\{d_{i m}, \operatorname{cap}_{s}\right\}$ which is the minimum of the expected demand for movie $m$ on time point $i$ and the capacity of screen $s$ if $(i, m)$ and $(j, n)$ are in the same layer, and $Q-\min \left\{d_{i m}, c a p_{s}\right\}$ if the nodes are in a different layers. The basic movie scheduling, where every movie can only be shown on one screen, can now be seen as finding a path from source to sink in each network such that the total cost of the paths is minimal and each movie is in exactly one of the paths. In mathematical terms, this can be directly formulated as a set partitioning problem with decision variable $x_{p}^{s}$, which is 1 if path $p$ is selected in network $G^{s}$ and 0 otherwise. 


$$
\begin{array}{cc}
\min \sum_{s \in S} \sum_{p \in P^{s}} c_{p} x_{p}^{s} & \\
\text { s.t. } \sum_{s \in S} \sum_{p \in P^{s}} a_{m p}^{s} x_{p}^{s}=1 & \forall m \in M \\
\sum_{p \in P^{s}} x_{p}^{s}=1 & \forall s \in S \\
x_{p}^{s} \in\{0,1\} & \forall s \in S, p \in P^{s}
\end{array}
$$

Herein, $P^{s}$ is defined as the set of all paths in $G^{s}$, and parameters $a_{m p}^{s}$ are 1 if movie $m$ is in path $p$ corresponding to screen $s$ and 0 otherwise. Notice that the formulation can be extended to the situation where a movie can be shown on different screens during the day. Since this situation is not the case in our application, we do not take it into consideration.

Of course, not all practical aspects have been taken into account in this mathematical formulation yet. In the remainder of this section, we discuss three important aspects that can be taken into account by adding extra sets of constraints.

The first aspect deals with the fact that only one movie can start at the same time on a floor during crowded periods. This can be formulated with the following set of constraints:

$$
\sum_{s \in S} \sum_{p \in P^{s}} b_{i p}^{s} e_{f}^{s} x_{p}^{s} \leq 1
$$

where $F$ is the set of floors and $T_{1}$ is the set of time points over which this condition should hold. Parameters $\mathrm{b}_{\mathrm{ip}}^{\mathrm{s}}$ and $\mathrm{e}_{\mathrm{f}}^{\mathrm{s}}$ are 1 if at time point $i$ a movie starts in path $p$ belonging to screen $s$ is, and screen $s$ is on floor $f$, respectively, and 0 otherwise.

As mentioned earlier, it is important for the management that in certain pre-specified intervals (in our case every 20 minutes), there is at least one movie starting. However, this extra requirement can result in a too large reduction of the number of visitors. Therefore, we add a $0 / 1$ decision variable $y_{l}$ indicating whether these constraints are satisfied or not (it is 1 if the constraint is not satisfied). The following set of constraints is added to the formulation:

$$
\sum_{s \in S} \sum_{p \in P^{s}}\left(b_{i p}^{s}+\ldots+b_{j p}^{s}\right) x_{p}^{s}+y_{l} \geq 1 \quad \forall l \in L, i, j \in T_{l}
$$


where $L$ is the set of intervals and $T_{l}$ are the time points in interval $L$. Moreover, we add the term $\sum_{l \in L} R y_{l}$ in the objective function, where $R$ is a penalty for violating one of these restrictions.

The third and final aspect that we explicitly take into consideration has to do with the closure of the theater. Since it is undesirable for all movies to end at the same time, in a certain fraction of $r$ screens the last movie has to be finished before a certain time:

$$
\sum_{s \in S} \sum_{p \in P^{s}} h_{p}^{s} x_{p}^{s} \geq r
$$

where $h_{p}^{s}$ is 1 if the last arc in path $p$ starts from a node $(i, m)$ where $t_{i}+d r_{m}$ is less than a certain prespecified time.

To summarize, the MSP can be formulated as follows:

$$
\begin{array}{cl}
\min \sum_{s \in S} \sum_{p \in P^{s}} c_{p} x_{p}^{s}+R \sum_{l \in L} y_{l} & \\
\text { s.t. } \sum_{s \in S} \sum_{p \in P^{s}} a_{m p}^{s} x_{p}^{s}=1 & \forall m \in M, \\
\sum_{p \in P^{s}} x_{p}^{s}=1 & \forall s \in S, \\
\sum_{s \in S} \sum_{p \in P^{s}} b_{i p}^{s} e_{f}^{s} x_{p}^{s} \leq 1 & \forall f \in F, i \in T_{1}, \\
\sum_{s \in S} \sum_{p \in P^{s}}\left(b_{i p}^{s}+\ldots+b_{j p}^{s}\right) x_{p}^{s}+y_{l} \geq 1 & \forall l \in L, i, j \in T_{l}, \\
\sum_{s \in S} \sum_{p \in P^{s}} h_{p}^{s} x_{p}^{s} \geq r, & \\
x_{p}^{s} \in\{0,1\} &
\end{array}
$$

\section{2 The Proposed Algorithm}

As argued before, we will use column generation techniques to deal with the large number of variables. The general idea behind column generation, introduced by Dantzig and Wolfe (1960), is to solve a sequence of reduced problems, where each reduced problem only contains a small portion of the set of variables (columns). After a reduced problem is solved, a new set of columns is obtained by using dual information of the solution. The column generation algorithm converges once it has established that the 
optimal solution based on the current set of columns cannot be improved upon by adding more columns. Then the optimal solution of the reduced problem is the optimal solution of the overall problem. We will refer to the reduced problem as the master problem and to the problem of generating a new set of columns as the pricing problem.

Traditionally, column generation for integer programs is used to solve their LP-relaxations. However, we will use it in combination with Lagrangian relaxation (for the reader unfamiliar with Lagrangian relaxation, see Fisher (1981)). This approach has been chosen for the following reasons.

- For this problem, a set of columns generated to compute the lower bound turns out to be a set from which we can select a reasonably good feasible solution.

- Since we compute a lower bound on the optimal solution, we obtain an indication about the quality of the constructed feasible solution.

- Lagrangian relaxation has been shown to provide tight bounds for set partitioning type of problems (see e.g. Beasley (1995)).

We refer to Huisman et al. (2005) for a more comprehensive discussion about combining column generation with Lagrangian relaxation. In the remainder of this section, we present the algorithm to solve the MSP. In Figure 2, we summarize the algorithm's main steps.

Step 0: Initialization

Construct an initial feasible solution and take those columns as starting ones.

Step 1: Master problem

Obtain a good set of dual multipliers by solving a Lagrangian dual problem.

Step 2: Pricing problem Generate for each screen new columns with negative reduced costs. As long as we find new columns, return to Step 1. Otherwise, go to Step 3.

Step 3: Construct a feasible solution Solve the corresponding integer program with the subset of columns generated in Step 2 and the initial ones from Step 0 to optimality.

\section{Figure 2: Schematic picture of the algorithm}

In Step 0, we construct an initial feasible solution by showing each movie once and setting all yvariables equal to 1 . This solution gives an initial set of columns. The first time that we solve the master problem in Step 1 we use this set columns. 


\section{Master problem}

To solve the master problem (Step 1), we use a relaxation of (MSP) by replacing the "=" signs by " $\geq$ " signs and subsequently relaxing the constraints in a Lagrangian way. That is, we associate Lagrange multipliers $\lambda_{m}, \mu_{s} v_{f i}, \sigma_{i j l}$ and $\omega$ to Constraints (9-13), respectively. The remaining Lagrangian subproblem can then be rewritten as:

$$
\begin{array}{cll}
\min \sum_{s \in S} \sum_{p \in P^{s}} \bar{c}_{p}^{s} x_{p}^{s}+\sum_{l \in L}\left(R-\sigma_{i j l}\right) y_{l} & \\
\text { s.t. } & x_{p}^{s} \in\{0,1\} & \forall s \in S, p \in P^{s}, \\
y_{l} \in\{0,1\} & \forall l \in L,
\end{array}
$$

where $\bar{c}_{p}^{s}=c_{p}-\sum_{m \in M} \lambda_{m} a_{m p}^{s}-\mu_{s}-\sum_{f \in F} \sum_{i \in T_{1}} v_{f i} b_{i p}^{s} e_{f}^{s}-\sum_{l \in L} \sum_{i \in T_{1}} \sum_{j \in T_{1}} \sigma_{i j l}\left(b_{i p}^{s}+\ldots+b_{j p}^{s}\right)-\omega$

The Lagrangian subproblem can be solved by inspection, i.e. $x_{p}^{s}=1$ if $\mathrm{c}_{\mathrm{p}}^{-\mathrm{s}}<0$ and 0 otherwise, and $y_{l}=1$ if $R<\sigma_{i j l}$ and 0 otherwise. In this way, we obtain a lower bound for the given set of columns. We apply subgradient optimization to find the best lower bound. For the reader interested in this topic, please see, for example, the survey of Beasley (1995). We will not go to into further detail here.

\section{Pricing problem}

In Step 2 we generate new columns with negative reduced costs, where the reduced costs of a

column corresponding to path $p$ on screen $s$ is denoted by $c_{p}^{-s}$. For each screen, we generate new paths by solving an all-pair shortest path problem between each pair of nodes (excluding the source and sink node). From all these possible paths, we add the k-smallest ones to the master problem. By solving an allpair shortest path problem, we get a large variety in columns, which makes it possible that only a few iterations in the column generation procedure are necessary. Furthermore, we calculate a lower bound on the overall problem by adding the reduced costs of all generated columns by the lower bound obtained in Step 1. If this difference is small enough, we quit the column generation procedure and construct a feasible solution. Otherwise, we return to Step 1. 


\section{Feasible solution}

Finally, we construct a feasible solution for the movie scheduling problem. This is done by solving the problem (MSP) with the initial columns and the ones generated during the column generation to optimality. For this purpose, we use the commercial MIP solver Cplex 7.1.

\subsection{Illustrative Computational Results}

To help evaluate the algorithm's performance we conducted several tests on Pathé's data. We constructed daily schedules for a number of days, with varying numbers of movies. After discussing the results with Pathé, we set the values for the parameters Q and R (penalties) to 100 and 10, respectively.

\begin{tabular}{|c|c|c|c|c|c|c|}
\hline & $\begin{array}{c}\text { \# mov- } \\
\text { ies }\end{array}$ & Gap (abs) & Gap (in \%) & Cpu* Step 1 & Cpu Step 2 & Total cpu** \\
\hline 20050303 & 19 & 42 & 1.34 & 60 & 65 & 145 \\
\hline 20050310 & 18 & 23 & 1.05 & 42 & 50 & 116 \\
\hline 20050317 & 17 & 27 & 1.40 & 87 & 64 & 185 \\
\hline 20050324 & 19 & 30 & 1.52 & 56 & 54 & 117 \\
\hline 20050407 & 18 & 28 & 1.32 & 56 & 46 & 110 \\
\hline 20050414 & 16 & 33 & 2.04 & 49 & 44 & 101 \\
\hline 20050421 & 18 & 18 & 1.00 & 86 & 75 & 176 \\
\hline 20050428 & 17 & 18 & 1.59 & 44 & 46 & 117 \\
\hline 20050505 & 22 & 25 & 3.27 & 105 & 215 & 441 \\
\hline 20050519 & 17 & 28 & 2.67 & 62 & 61 & 138 \\
\hline 20050526 & 15 & 6 & 0.60 & 43 & 27 & 74 \\
\hline 20050602 & 15 & 22 & 1.36 & 58 & 34 & 98 \\
\hline 20050630 & 19 & 23 & 1.36 & 44 & 56 & 107 \\
\hline 20050714 & 20 & 21 & 1.52 & 102 & 107 & 223 \\
\hline Average & 18 & 24.57 & 1.58 & 64 & 67 & 153 \\
\hline
\end{tabular}

* The Cpu times are measured in seconds on a Pentium III, 1 GHz PC (384MB RAM);

** Total cpu time also includes Step 0 and 3

Table 1: Computational results of the movie scheduling algorithm

In Table 1, we report for 14 instances (days) the number of movies, the absolute and relative gap between upper and lower bounds on the optimal solution, and the computation times (in seconds) for the 
master problem (Step 1), pricing problem (Step 2) and the complete algorithm. The number of screens is equal to 13 for all instances.

As can be seen in Table 1, the relative gap is between 0.60 and $3.27 \%$. The average gap is $1.58 \%$. The total computation time is very reasonable: on average it takes about 2.5 minutes. The most time, as expected, is spent in the column generation part of the algorithm, where the time to solve the master and pricing problem are almost equal.

\section{The Conditional Forecasting Method}

Developing a week's schedule requires forecasts for $A_{j t}$, the attendance for a screening of movie $j$ starting at time $t$, for all movies available for screening and for all possible times (in ten minute intervals) for every day of that week. Such forecasts would be required each Sunday to be available for the optimization algorithm so that recommendations to management could be made on Monday. In order to use the most recent possible data, the forecasting procedure had to be completed in less than 12 hours. This essentially ruled out some simulation-based forecasting models such as Hierarchical Bayes models (e.g., Ainslie, Drèze and Zufryden 2005).

As is well known, the age of a movie is an important factor in determining its appeal to the audience. A number of empirical studies report that moviegoers' demand for most movies typically decreases across weeks (e.g., Sawhney and Eliashberg 1996, Krider and Weinberg 1998, Lehmann and Weinberg 2000). We use a two-parameter exponential model to capture these effects. For most movies, these parameters can be estimated from the detailed attendance records maintained by Pathé. For movies for which we do not have attendance data for the De Munt Theater for at least two weeks, we developed a secondary procedure to estimate the two parameters of the exponential model. This procedure is described more fully below. Also, there are other effects, such as holidays, which may influence attendance and we include control variables for such effects as well.

In addition to these weekly effects, there are more micro effects that need to be considered. In particular, we introduce variables that account for day of the week and time within a day (in hourly inter- 
vals) at which movies are shown. We also allow for other detailed factors such as weather (temperature, precipitation) and whether the Dutch national football (soccer) team is playing a major international match. Our choice of variables to include was guided by preliminary analysis of the data and published papers. As described below, we divided our procedure into three steps.

\subsection{Method description}

\section{$\underline{\text { Step 1: Demand Model Estimation }}$}

In the first step, we use past attendance figures to estimate a demand model, which separates out different time-varying patterns. Formally, we model $A_{j t}$, the attendance for a showing of movie $j$ starting at time $t$ as:

$$
\begin{gathered}
A_{j t}=\exp \left(\sum_{\forall j} \theta_{j} \cdot I_{\{j\}}+\lambda_{j} \cdot A G E_{j t}\right. \\
+\sum_{\forall h} \beta_{h} \cdot I_{\{h\} t}+\sum_{\forall d} \omega_{d} \cdot I_{\{d\} t}+\sum_{\forall v} \gamma_{v} \cdot I_{\{v\} t}+\delta_{S A T N I G H T}: S A T N I G H T_{t}+\delta_{S U N P M} S U N P M_{t} \\
\left.+\delta_{N G} \cdot N G_{t}+\delta_{D T E M P} \cdot D T E M P_{t}+\delta_{D P R E C I P} \cdot D P R E C I P_{t}+\varepsilon_{j t}\right)
\end{gathered}
$$

where

$I_{\{j\}} \quad \equiv$ Indicator variable for the event that movie $j$ is being shown

$A G E_{j t} \equiv$ Age of movie $j$ at time $t$ (number of weeks since movie $j$ 's first regular showing at the theater)

$I_{\{h\} t} \quad \equiv$ Indicator variable for the event that starting time $t$ is within hour $h, h \in\{10 \mathrm{am}-10 \mathrm{pm}\}$

$I_{\{d\} t} \quad \equiv$ Indicator variable for the event that starting time $t$ is on day $d, d \in\{$ Monday, Tuesday, ..., Sunday $\}]^{4}$

$I_{\{v\} t} \quad \equiv$ Indicator variable for the event that starting time $t$ is on an Amsterdam holiday or school vacation $v, v \in\{$ Spring vacation, May vacation, Ascension Day, Whit Sunday, Whit Monday, Easter weekend, Summer vacation, Fall vacation, Christmas vacation $\}$

$S_{A T N I G H T_{t}} \equiv$ Indicator variable for the event that starting time $t$ is between $11 \mathrm{pm}$ and $1 \mathrm{am}$ of a Saturday

$S U N P M_{t} \quad \equiv$ Indicator variable for the event that starting time $t$ is between $2 \mathrm{pm}$ and $6 \mathrm{pm}$ of a Sunday

$N G_{t} \quad \equiv$ Indicator variable for the event that starting time $t$ occurs during the time when the Dutch national team is playing in a major tournament and is televised

$D T E M P_{t} \quad \equiv$ The maximum temperature of the day starting time $t$ is in

DPRECIP $_{t} \equiv$ The indicator variable for the event that starting time $t$ is in a day with nonzero precipitation (e.g., rain or snow).

\footnotetext{
${ }^{4}$ For completeness, all possible values of the indicator variable are listed in the text. The base case for all the indicator variables are stated in the tables reporting results.
} 
$\theta_{j}$ and $\lambda_{j}$ are the two movie-specific parameters capturing the time trend of an individual movie's attractiveness across weeks. In particular, we assume the attractiveness of each movie title follows an exponential decay, with $\theta_{j}$ characterizing the scale of the movie's attractiveness (opening strength) and $\lambda_{j}$ capturing the weekly decay of the attractiveness.

As discussed earlier, the other type of time trends inherent in the attendance is moviegoers' time preference to watch a movie; these effects are captured by three sets of parameters, $\beta_{h}, \omega_{d}$, and $\gamma_{v}$. The $\gamma_{v}$ 's capture the time of year effect. In particular, as moviegoers tend to have more free time for leisure activities such as going to movie theaters during holidays or school vacations, as compared to normal work or school days, we expect all of these parameters to be positive. Five school vacation periods, namely spring, May, summer, fall and Christmas vacations are first identified. Three public holidays outside these vacation periods, namely Ascension Day, Whit weekend and Easter weekend are then added to capture the other potential holiday effects.

The day of week effect is captured by the $\omega_{d}$,'s. Seven parameters $\omega_{M O N}, \omega_{T U E}, \omega_{W E D}, \omega_{T H U}, \omega_{F R I}$, $\omega_{S A T}$ and $\omega_{S U N}$ capture this time trend. At the more micro level, the $\beta_{h}$ 's capture the time of day effect. We expect the $\beta_{h}$ 's corresponding to the daytime to be smaller than those for the evening. While the normal operating hours of the DeMunt are bound by 11 pm, occasionally some movies are shown after 11 pm on Saturday. We therefore use the parameter $\delta_{S A T N I G H T}$ to capture the effect of this extension on Saturday. We also include a parameter to represent Sunday afternoons $\left(\delta_{S U N P M}\right)$ as our preliminary data analysis showed that attendance for this day was typically higher in the afternoon than on other days.

We want to control in our demand model three additional systematic shifts of the attendance: whether the movie is screened against a strong competing leisure activity (we focus on major tournament soccer game played by Dutch national team), the highest temperature of the day, and whether there is rain or snow during the day. The parameters, $\delta_{N G}, \delta_{D T E M P}$ and $\delta_{D P R E C I P}$ capture these systematic shifts.

After assuming $\varepsilon_{j t} \sim \mathrm{N}\left(0, \sigma^{2}\right)$, we take the logarithms of (18) and estimate the transformed model by OLS, using all the available attendance figures up to the Sunday preceding the Thursday the new mov- 
ie program starts. When additional attendance data are added each week, the demand model is reestimated with the extended data set.

The current demand model assumes that the attendance of movie $j$ starting at time $t$ is not affected by which specific movies are shown at the same time. More complex models that capture substitution effects are explored in Ho (2005), but they provide limited improvement in the prediction power, while greatly increasing the complexity of the mathematical optimization. Consequently, we retained (18) as the demand model for the current project.

\section{$\underline{\text { Step 2: Determination of Movie-Specific Parameters }}$}

In capturing individual movies' attractiveness, the two sets of movie-specific parameters, $\theta_{j}$ and $\lambda_{j}$ are crucial to our demand forecasting. We separate our forecasting procedure into two cases: (1) movies with attendance data for 2 or more weeks and (2) newly released movies with no data, as they are yet to be shown, or with one week of data, if they have been shown for just one week at the De Munt Theatre. When there are two or more weeks of data for a movie title (case 1), we have sufficient information to estimate both $\theta_{j}$ and $\lambda_{j}$ from (18). To make forecasts for such a movie, we use the estimates of $\theta_{j}$ and $\lambda_{j}$, obtained from the first step with the most recent data. On the other hand, for movies with either limited or no attendance data (case 2), there are no estimates of $\theta_{j}$ (and/or $\lambda_{j}$ ) from the first step. To deal with this issue, we first built a regression model that relates $\theta_{j}\left(\right.$ and/or $\left.\lambda_{j}\right)$ to movie attributes that might explain $\theta_{j}\left(\right.$ and/or $\left.\lambda_{j}\right)$. Once we obtain the coefficients of this regression model, we use these numbers and the values of the attributes for a new movie to estimate that movie's value of $\theta_{j}$ (and/or $\lambda_{j}$ ).

More specifically, we regress (say) the values of $\hat{\theta}_{j}$, the estimates of $\theta_{j}$ from the first step on various movie attributes using the following model:

$$
\begin{aligned}
& \hat{\theta}_{j}=\rho_{0}+\rho_{O U} \cdot O-U S A_{j}+\rho_{O D} \cdot O-D U T C H_{j}+\rho_{O O} \cdot O-O T H E R_{j} \\
& +\rho_{L E} \cdot L-E N G L I S H_{j}+\rho_{L D} \cdot L-D U T C H_{j}+\rho_{L O} \cdot L-O T H E R_{j}+\rho_{D D} \cdot D-D U T C H_{j} \\
& +\rho_{S Q} S_{E Q U U E L_{j}}+\rho_{F C} \cdot F R A N C H I S E_{j}
\end{aligned}
$$




$$
\begin{aligned}
& +\rho_{B O W 1} \cdot B O W 1_{j}+\rho_{T N W 1} \cdot T N W 1_{j}+\rho_{B O W 1 W 2} \cdot B O W 1 W 2_{j} \\
& +\rho_{G} \cdot M P A A-G_{j}+\rho_{P G} \cdot M P A A-P G_{j}+\rho_{P G 13} \cdot M P A A-P G 13_{j}+\rho_{R} \cdot M P A A-R_{j} \\
& +\rho_{D R A} \cdot D R A M A_{j}+\rho_{A C T} \cdot A C T I O N_{j}+\rho_{C O M} \cdot C O M E D Y_{j}+\rho_{R C O M} \cdot R C O M E D Y_{j} \\
& +\rho_{M U S} \cdot M_{U S I C A L_{j}}+\rho_{S U S} S U S P E N S E_{j}+\rho_{S F} \cdot \text { SCIFI }_{j}+\rho_{\text {HOR }} \cdot \text { HORROR }_{j}+\rho_{\text {FAN }} \cdot \text { FANTASY }_{j}+\rho_{W E S} \cdot \text { WESTERN }_{j} \\
& +\rho_{A N I} \cdot A_{N I M A T E D}+\rho_{A D V} \cdot A D V E N T U R E_{j}+\rho_{D O C} \cdot D O C U_{j}+\varepsilon_{j}^{\prime}
\end{aligned}
$$

See Appendix 1 for definitions of these characteristics. For Dutch and other movies for which there was no U.S. release, such variables as US box office revenues are set to zero. The indicator variable is sufficient to capture the fact that no additional information from the US box office is available for estimation purposes.

Using the parameter estimates, we then predict $\theta_{j}$ for the new movies yet to be opened (case 2) by inserting the new movies' characteristics into (19). (For the few movies that opened simultaneously in the US and the Netherlands, outside estimates of the US box office were used.) For movies with one week of data, a similar procedure is used for the decay rate, $\lambda_{j}$.

\section{Step 3: Attendance Forecasts}

In this step, we use (18) to generate forecasts for all movies available for screening (cases 1-2) at all possible times in the new movie program. Specifically, we take all the parameter estimates from the first step and, when needed, the estimates of $\theta_{j}$ and $\lambda_{j}$ from the second step, to forecast the expected attendance for movie $j$ starting at future time $t, E\left(A_{j t}\right)$ :

$$
\begin{gathered}
E\left(A_{j t}\right)=\exp \left(\sum_{\forall j} \hat{\theta}_{j} \cdot I_{\{j\}}+\hat{\lambda}_{j} \cdot A G E_{j t}\right. \\
+\sum_{\forall h} \hat{\beta}_{h} \cdot I_{\{h\} t}+\sum_{\forall d} \hat{\omega}_{d} \cdot I_{\{d\} t}+\sum_{\forall v} \hat{\gamma}_{v} \cdot I_{\{v\} t}+\hat{\delta}_{\text {SATNIGHT }} \cdot S A T N I G H T_{t}+\hat{\delta}_{\text {SUNPM }} \cdot S U N P M_{t} \\
\left.+\hat{\delta}_{N G} \cdot N G_{t}+\hat{\delta}_{D T E M P} \cdot D T E M P_{t}+\hat{\delta}_{D R E C I P} \cdot D P R E C I P_{t}+\hat{\sigma}^{2} / 2\right)
\end{gathered}
$$

As our parameter estimates are obtained by taking the logarithms of (18) and then estimating the transformed model with OLS, when we use these OLS estimates in the original non-linear model of (18), there 
will be a downward bias in the forecasts (Hanssens et al 2003, p.395). We use the correction factor $\hat{\sigma}^{2} / 2$ in (20) to compensate for this downward bias $\left(\hat{\sigma}^{2}\right.$ is the estimate for the variance of $\varepsilon_{j t}$ in $\left.(18)\right)$.

Unlike other variables in (20), the future values of the two weather variables $\left(D T E M P_{t}\right.$ and $\left.D P R E C I P_{t}\right)$ are unknown at the time of forecasting. We therefore use the government weather forecasts for Amsterdam, where the De Munt is located, as the values for $D T E M P_{t}$ (=forecasted maximum temperature $)$ and $\operatorname{DPRECIP}_{t}(=1$ if probability of precipitation $>0.5)$.

Data Management

For our runs of SilverScheduler in the 14 weeks in the period March-July 2005, we continuously updated our estimates as new data became available. Specifically, we started with 57 weeks (January 29, 2004 - February 27, 2005) of attendance data, giving us 23,148 observations for estimation in the first week. For each Sunday, the data set was updated with a new week of data up to that day, adding around 400 new observations (each week has about 400 movie screenings) ${ }^{5}$.

Some particular aspects of our data collection process are worth noting. While there were some free admissions to movies, our estimation only included paid admissions. As only $3 \%$ of movies reached $90 \%$ of capacity and average utilization per showing was about $26 \%$, we do not consider capacity constraints in either our estimation or programming model. Also, we do not consider price effects. Although the De Munt theater charges slightly different prices for showings starting in different time slots (e.g., matinees) and for moviegoers of different ages (e.g., senior discounts), prices are the same across different movie titles. As a result, the price variation is perfectly correlated with the time of day variation. Therefore, the price effect will be completely absorbed by the time of day parameters and there is no need to include any price variables.

\footnotetext{
${ }^{5}$ After completion of the test runs, we performed an audit of our data management process and found some minor data management problems. For example, the attendance at a movie that was double booked in two screens was combined into one data point. These effects had a small overall impact and led to less accurate forecasts than otherwise would have occurred. (We verified this by running our model for an additional week after discovering these problems and found an increase in the $\mathrm{R}^{2}$ between forecasts sales and attendance compared to our mean results.) In this paper, we use the forecasts originally generated as they were the ones used in our interactions with management.
} 


\subsection{Evaluation of Forecasting Performance}

Table 2 shows the estimation results for our main forecasting model (18). For brevity, we report only the estimation results in the first week. To identify the model (18), we set "classics" screened at 8pm on Saturday as the base case. As can be seen in Table 2, the results demonstrate high face validity and strong explanatory power. The $\mathrm{R}^{2}$ for the first week is 0.64 ; across the 14 weeks in our study, the $\mathrm{R}^{2}$ between predicted attendance and actual attendance averaged 0.65 , with a high value of 0.81 in week 6 and a low value of 0.43 in week 8 .

As can be seen in Table 2, all but one of the estimated coefficients are statistically significant; this is not surprising given the large sample size. More importantly, the values of the coefficients are consistent with expectations: 1) the estimates for $\beta_{h}$ increase as we go into the evening ( $8 \mathrm{pm}$ is the most preferable time to watch a movie), 2) the estimates for $\omega_{d}$ on Saturday and Sunday are the largest; 3) all the estimates for $\gamma_{v}$ are significantly positive; 4) Sunday afternoons are better than those of other days (but other times on Sundays are equivalent to the base case); and 5) national soccer games negatively affect movie attendance. In addition to the coefficients reported in Table 2, movie specific parameter values for $\theta_{j}$ and $\lambda_{j}$ were obtained for each movie. For example, based on the data available for the week of March 3 , the movie Passion of the Christ had values of $\theta_{j}=0.224$ and $\lambda_{j}=-0.053$, indicating a strong opening and a slow decay rate. For movies in their first or second week of showing, the characteristics of the movies were used to estimate values of $\theta_{j}$ and $\lambda_{j}$ as shown in equation (19). As expected, the need to rely on estimates had an effect on the accuracy of our forecasts. For example, across the 14 weeks, the average error for new movies was -19.7 admissions per showing; for movies with just one week of data, the mean error was 12.8 admissions per showing; and for movies with two or more weeks of data, the mean error was -6.4 admission tickets per showing ${ }^{6}$. Future work should examine methods to obtain better estimates for new movies. Market research techniques such as those described in Eliashberg et al. (2000) or data from new data sources such as the Hollywood Stock Exchange (Spann and Skiera 2003) could be usefully employed to obtain such results. 


\begin{tabular}{|l|r|r|l|r|r|}
\hline & Movie Program: Mar 3-9, 2005 & & & \\
\hline R-Square & 0.6423 & & TUE & -0.380 & $<.0001$ \\
\hline Adj R-Sq & 0.6361 & & WED & -0.431 & $<.0001$ \\
\hline $\mathrm{N}$ & 23148 & & THU & -0.291 & $<.0001$ \\
\hline Variable & Parameter Estimate & Pr $>|\mathrm{t}|$ & FRI & -0.165 & $<.0001$ \\
\hline Intercept & 5.071 & $<.0001$ & SUN & -0.015 & $<.4333$ \\
\hline $10 \mathrm{am}$ & -2.011 & $<.0001$ & SATNIGHT & -1.033 & $<.0001$ \\
\hline $11 \mathrm{am}$ & -1.712 & $<.0001$ & SUNPM & 0.512 & $<.0001$ \\
\hline $12 \mathrm{pm}$ & -1.885 & $<.0001$ & National Soccer & -1.123 & $<.0001$ \\
\hline $1 \mathrm{pm}$ & -1.334 & $<.0001$ & Easter Weekend & 0.452 & $<.0001$ \\
\hline 2pm & -1.063 & $<.0001$ & Ascension Day & 0.891 & $<.0001$ \\
\hline $3 \mathrm{pm}$ & -1.066 & $<.0001$ & Whit Weekend & 0.850 & $<.0001$ \\
\hline 4pm & -1.164 & $<.0001$ & Spring Vacation & 0.589 & $<.0001$ \\
\hline $5 \mathrm{pm}$ & -1.063 & $<.0001$ & May Vacation & 0.365 & $<.0001$ \\
\hline $6 \mathrm{pm}$ & -0.768 & $<.0001$ & Summer Vacation & 0.142 & $<.0001$ \\
\hline $7 \mathrm{pm}$ & -0.349 & $<.0001$ & Fall Vacation & 0.616 & $<.0001$ \\
\hline 9pm & -0.237 & $<.0001$ & Xmas Vacation & 0.723 & $<.0001$ \\
\hline $10 \mathrm{pm}$ & -0.491 & $<.0001$ & Daily Max. Temp. & -0.003 & $<.0001$ \\
\hline MON & -0.590 & $<.0001$ & Precipitation & 0.084 & \\
\hline
\end{tabular}

Base Case: Classics screened at 8pm on Saturday

Table 2: Estimation Results of Demand Model (18)

\section{Application of SilverScheduler to a Multiplex in Amsterdam}

\subsection{Empirical setting}

The complete SilverScheduler procedure (scheduling algorithm + conditional forecasting method) was applied to the De Munt Theater in downtown Amsterdam. We had data for 14 different movie weeks for the De Munt, as well as data from the previous year for estimating initial values of the model parameters. For each of the 14 weeks we received the following information from Pathé:

- The movies to be scheduled in that particular week (i.e., the list of movies), with their running times. The average number of movies to be scheduled was 18, ranging from 15 to 22 .

- Contractual agreements with distributors that certain movies will be shown in certain screening rooms. Typically, such agreements are made for newly released movies (1 to 3 per week). Sometimes the screening room is specified; sometimes the agreement says that the screening room should be above a specific seat capacity.

\footnotetext{
${ }^{6}$ The Mean Absolute Errors were also calculated and were 37.2, 22.6, and 19.0 respectively for new movies, movies with one
} 
- Opening and closing times of the theater for weekdays and weekend days and information about the times required for cleaning the screening rooms between two shows.

\section{Additional managerial requirements}

First, movie visitors should never have to wait more than 20 minutes until the next movie starts. In this way, Pathé tries to appeal to people who just want to see a movie, without a strong preference for a particular one. Therefore, the time between two subsequent movie starts should not be more than 20 minutes (implemented with a penalty function). Second, for high potential movies there should be the possibility of double of even triple booking. This means that the same movie is shown in different screening rooms at the same time. We accommodated this in our system, and to get the most out of such high potential movies, we decided that the starting times of the same movie in different screening rooms would be at least one hour apart. Management informed us as to which movies should be double or triple booked. Third, there is a problem of crowding. The screening rooms of De Munt are on two different floor levels, with rooms 1 to 8 on one floor and rooms 9 to 13 on the other. The corridors and hallways are quite narrow. To avoid overcrowding, a rule was introduced that at the busy times (all evenings and Saturday and Sunday afternoons) not more than one movie can start in the same time block on the screens 1 to 8 , and the same for the screens 9 to 13 . This spread in movie starts is also favorable for the sales from the concession counters, which have a more evenly distributed demand in this way. Fourth, management wanted to limit the numbers of screen changes, i.e. the number of times that another movie has to be mounted on the projection system for a particular room. Movie reels are physically quite big and difficult to handle, and. there is a (small) probability that the movie reel will fall and be damaged. We limited the number of screen changes by imposing a penalty for each screen change.

As demand input we used the estimates produced by the demand forecasting procedure, as described earlier. This means that we have a forecast for the number of visitors for every movie for each possible starting time/day combination in the week for which the schedule is produced. Here we work with a grid of starting times that are one hour apart. 


\section{From day schedules to a week schedule}

Employing the procedures described above, we used SilverScheduler to make schedules for each of the 14 weeks in our dataset. As is clear from the description in section 3, the scheduling algorithm, in principle, makes a schedule for a day (it solves the MSP). However, De Munt does not have the same movie schedule on each of the seven days of the week. First of all, Saturdays and Sundays are different from weekdays. On both weekend days the theater opens at 10 am (compared to noon on weekdays) and on these days there are some children movies shown (until $6 \mathrm{pm}$ ). Furthermore, Saturday is different from Sunday, because Saturday is the only day that the theater is open until $1.30 \mathrm{am}$. On all other days, it closes at midnight. Finally Wednesday is different from the other weekdays, since children movies are shown on Wednesday afternoons. To take these differences into account, we proceeded as follows. We first applied SilverScheduler to generate a schedule for the four days (Thursday, Friday, Monday, and Tuesday) that have the same schedule. Subsequently this "base schedule" served as the starting point to make schedules for the other three days. Here the movies remain in the same screening room as in the base schedule, but the times are adapted according to the requirements of the specific days. Furthermore, on Saturday, Sunday, and Wednesday children's movies were inserted during the day, replacing those movies of the base schedule which had the lowest number of visitors.

\subsection{Results for one day}

To illustrate the SilverScheduler approach to movie scheduling and to compare it to the corresponding actual Pathé schedule, constructed manually, we use the first day (Thursday, March 3) in our dataset. There are 26 movies in total for this week, including 2 copies of Constantine. (For a list of movies and name abbreviations, see Appendix 2.) The last 3 movies listed are for dedicated showings in specific screens. There are 4 children movies (not shown on Thursdays, Fridays, Mondays or Tuesdays), so there remain 19 movies that need to be scheduled. 
Figure 3 shows (1) the actual schedule as produced by Pathé management (left), and (2) the schedule generated by SilverScheduler (right). As can be seen from Figure 3, we have divided the opening time of the theatre [from 12.00 (noon) to 00.00 (midnight)] into time intervals of 10 minutes each. The numbers behind the movie names are the forecasted numbers of visitors for the particular show. For this particular week there was only one contractual agreement with a distributor, requiring that the movie Hide and Seek (HS) should be shown in one of the two largest screening rooms, i.e., either in Screening Room 3 (340 seats) or in Screening Room 11 (382 seats). From Figure 3 it can be seen, that Pathé shows HS in Screening Room 11, whereas SilverScheduler puts it into Screening Room 3. In Figure 3 the differences in running times between the different movies are clear.

Manual Schedule

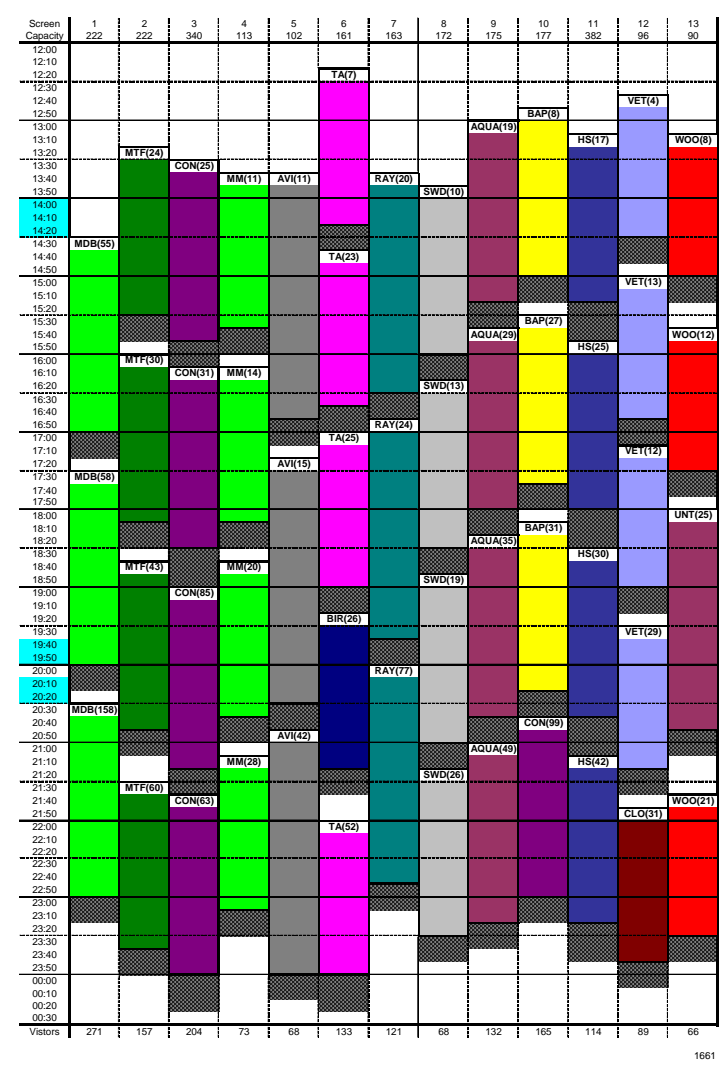

Schedule constructed with SilverScheduler

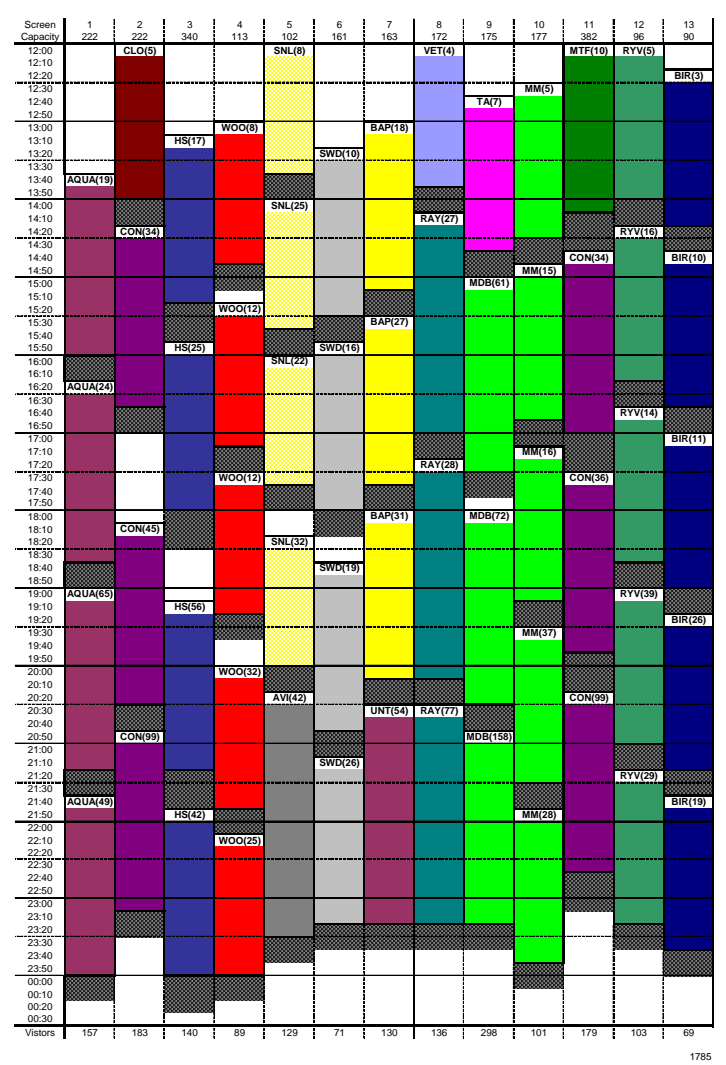

Figure 3: Schedules for Thursday March 3, 2005

For example, for Der Untergang (UNT) the running time is 165 minutes, and for Vet Hard (VET) 105 minutes. (Running times include advertising and trailers.) 
After each movie showing, there is a cleaning time before a new movie starts. The cleaning time is 20 minutes for a small room and 30 minutes for a large room.

\section{Evaluation of the solution}

The number of visitors for Thursday March 3, 2005, is 1785 in the SilverScheduler schedule, against 1661 in the manual schedule. For this day, SilverScheduler generates 124 extra visitors. SilverScheduler manages to schedule more shows on this particular day: 57, against 51 shows in the manually constructed schedule. So in this case, notwithstanding the extensive set of constraints, SilverScheduler is able to accommodate more shows. The SilverScheduler solution obeys the requirement that at least every 20 minutes a new movie should start. It is quite difficult to reach this goal when the schedule is made by hand. In the schedule for March 3 (Figure 3, left) this requirement is violated several times. For example, there are no movies that start between $13.50 \mathrm{~h}$ and $14.30 \mathrm{~h}$, between $19.30 \mathrm{~h}$ and $20.00 \mathrm{~h}$, and between $20,00 \mathrm{~h}$ and $20.30 \mathrm{~h}$. Also the other managerial requirements are violated repeatedly in the manual sche-

dule. For example, on the Saturday of the first week of our dataset (March 5, 2005; not shown here) several times the manual schedule allowed more than one movie to start on the same floor at the same time. This will produce undesirable crowding. Also, it occurred that there were more than two different movies in the same screening room on the same day, which violates the constraint of no more than two different movies on one screen (i.e., too much screen switching).

\subsection{Results for the 14 weeks}

We evaluate our overall results in several ways. First, we look at the scheduling efficiency, particularly the number of movies that are shown in each week. Second, we examine the revenue implications. Table 3 shows the number of movies ("movie showings") that would be shown in the Silver Scheduler schedules as compared to the Pathé schedules. Although SilverScheduler has to take into account a large number of constraints, it still manages to schedule slightly more movies than the programmers at 
Pathé have done $(+0.51 \%)$. But of course, the main contribution of SilverScheduler should be that it schedules "better" movies, which should result in higher ticket sales.

\begin{tabular}{|l|l|cc|cc|c|}
\hline & & \multicolumn{2}{|c|}{ Pathe schedule } & \multicolumn{3}{c|}{ SilverScheduler } \\
& & Movie Showings & Ticket sales & Movie Showings & Ticket sales & Improvement \\
\hline $\mathbf{2 0 0 5 0 9}$ & Mar 03 - Mar 09 & 394 & 18316 & 423 & 22930 & $25.2 \%$ \\
$\mathbf{2 0 0 5 1 0}$ & Mar 10 - Mar 16 & 396 & 13903 & 397 & 16379 & $17.8 \%$ \\
$\mathbf{2 0 0 5 1 1}$ & Mar 17 - Mar 23 & 415 & 15229 & 418 & 16069 & $5.5 \%$ \\
$\mathbf{2 0 0 5 1 2}$ & Mar 24 - Mar 30 & 424 & 22292 & 414 & 24053 & $7.9 \%$ \\
$\mathbf{2 0 0 5 1 4}$ & Apr 07 - Apr 13 & 410 & 14378 & 418 & 15531 & $8.0 \%$ \\
$\mathbf{2 0 0 5 1 5}$ & Apr 14 - Apr 20 & 414 & 12166 & 432 & 13240 & $8.8 \%$ \\
$\mathbf{2 0 0 5 1 6}$ & Apr 21 - Apr 27 & 429 & 15370 & 434 & 16614 & $8.1 \%$ \\
$\mathbf{2 0 0 5 1 7}$ & Apr 28 - May 04 & 416 & 13675 & 404 & 14138 & $3.4 \%$ \\
$\mathbf{2 0 0 5 1 8}$ & May 05 - May 11 & 439 & 9977 & 412 & 10502 & $5.3 \%$ \\
$\mathbf{2 0 0 5 2 0}$ & May 19 - May 25 & 412 & 9257 & 425 & 10887 & $17.6 \%$ \\
$\mathbf{2 0 0 5 2 1}$ & May 26 - Jun 01 & 406 & 9675 & 385 & 10099 & $4.4 \%$ \\
$\mathbf{2 0 0 5 2 2}$ & Jun 02 - Jun 08 & 418 & 11305 & 407 & 11639 & $3.0 \%$ \\
$\mathbf{2 0 0 5 2 6}$ & Jun 30 - Jul 06 & 409 & 13800 & 419 & 15654 & $13.4 \%$ \\
$\mathbf{2 0 0 5 2 8}$ & Jul 14 - Jul 20 & 419 & 15086 & 443 & 17763 & $17.7 \%$ \\
\hline
\end{tabular}

\section{Table 3: Comparison of the schedules of Pathé and SilverScheduler over all 14 weeks;} Visitors’ predictions based on predicted demand, using Equation (18).

It is clear from Table 3 that there is considerable improvement in attendance. If we compare both schedules, the Pathé schedules and those generated by SilverScheduler, and use in both cases Equation (18) for predicting the numbers of visitors, we see that SilverScheduler shows an increase of $10.83 \%$. This improvement reflects the contribution of the scheduling algorithm, given the demand (i.e. the predicted numbers of visitors). In other words, this is the contribution of SilverScheduler with perfect forecasts. However, actual visitor numbers tend to deviate from forecasted numbers. As we saw earlier, the correlation between actual and forecast is on average 0.65 . Of course, with imperfect forecasts, the improvement obtained through SilverScheduler will be less. To investigate this, we have also carried out a comparison of the schedules of Pathé and SilverScheduler based on the actual demand, as manifest from the observed visitor data (i.e. ex post). For this purpose, using the actual visitor data for the week, a regression model was estimated explaining the number of visitors per show, by the movie, the day of the week and the hour of the day. This model was then used to predict the number of visitors per show, for the schedule of Pathé, as well as the one from SilverScheduler. (For the Pathé schedule we could also have used the actual data directly, but we felt that using the ex-post regression model predictions in both 
cases, gives a fairer comparison). Using this approach, the estimated improvement in number of visitors was $2.53 \%$ over the 14 weeks. While lower than the level reported in Table 3 , this is still a considerable increase; it indicates there would be about 20,000 additional visitors per year, or about $€ 150,000$ in extra revenue $(\$ 187,000)$.

In addition to generating more visitors, SilverScheduler also provides direct operational efficiency by automating the preparation of the weekly movie schedule. SilverScheduler saves a considerable amount of managerial time and effort. The current process is cumbersome, often generating a fair amount of management frustration. Furthermore, all the expressed management constraints are met. Taking these managerial constraints into account (every 20 minutes a new movie, less crowding) should over time have a positive effect on the numbers of visitors which is not taken into account in the current computations of additional revenue.

\section{Conclusions and further development}

We have developed an algorithm, SilverScheduler that schedules movies over the days of the week and the times of the day. This algorithm, which follows the column generation approach, is able to produce solutions in a reasonable amount of time (on average 2.5 minutes) and with very good accuracy (on average within $1.57 \%$ of the optimum). A forecasting module was developed where the numbers of visitors are forecasted using a model estimated on data from previous weeks.

We carried out the scheduling for the De Munt Theater in Amsterdam for 14 weeks in 2005. Comparing the SilverScheduler results with the manual schedules, SilverScheduler generated improved results. Within the constraints set by logistical and managerial considerations, on average SilverScheduler scheduled about the same numbers of shows as management did. SilverScheduler, however, schedules better movies, and also takes better account of the managerial requirements compared to the manual solution. Assuming accurate forecasts, SilverScheduler generates nearly $11 \%$ more visitors than the manual schedules. When we make the comparison on the basis of actual demand in the given week, the improve- 
ment in visitors through SilverScheduler is $2.5 \%$. The latter amounts to $\$ 187,000$ in extra revenue for the De Munt, on an annual basis.

Additional research should help to further improve SilverScheduler. The first priority is to increase the accuracy of the forecasts. As we have seen, better forecasts significantly improve the performance of SilverScheduler as a whole. One possibility is to combine the currently used box office data with market research data $f$ market. This will improve the quality of the forecasts, in particular for new movies. Furthermore, new methodologies have become available for the early prediction of the success of new movies (e.g. Eliashberg et al 2000). Another area to examine is the impact of movie scheduling on concession sales. At present Pathé management estimates a constant amount of concessions sales per visitor. However, concession sales may vary by the times when movies are shown and may be dependent on the amount of queuing that occurs. An extension in this regard would pose interesting technical challenges, but could lead to substantial profit improvements given the high margins on concession sales. Also, SilverScheduler has to be developed further in the direction of a decision support tool, so that it can easily be used by the theater manager. This includes a user-friendly interface, and intuitively-appealing screens that are easy to navigate.

Pathé Nederland, the owner of the De Munt Theater, considers the implementation of SilverScheduler as an interesting opportunity. In that case, they will not just use it for De Munt, but for movie scheduling for all of their 12 movie theaters in the Netherlands. Pathé Nederland has a planning and ticketing software system in place already, and SilverScheduler can be used to deliver its schedules as the inputs to this system.

While we describe our development of the SilverScheduler algorithm in one theater, the problem is a widespread one. There are 7000 theaters in the US and 10,000 in Europe, all faced with the same problem. Movie theater programming is a time-consuming activity, and as our example illustrates, the required managerial constraints are not always met. It is clear that the possibility to delegate at least part of this task to a decision support system is an important step forward. Theaters are increasingly moving from manual ticketing systems to computer based ticketing systems. This should facilitate the adoption of 
computer based decision support systems. Probably even more important to the future impact of systems such as SilverScheduler in the movie industry is the expected growth of digital cinema. When this occurs, movies will not be delivered to theaters on huge reels anymore, but on simple diskettes or electronically. This will make theaters much more flexible in their scheduling. In such a situation, the scheduling possibilities will multiply, making it even more important to have an algorithm like SilverScheduler as part of a decision support system to help management find the best schedule for its theaters.

\section{References}

Ainslie, A., X. Dreze, F. Zufryden. 2005. Modeling Movie Lifecycles and Market Share. Marketing Sci. 24 508-517.

Barnhart, C., E.L. Johnson, G.L. Nemhauser, M.W.P. Savelsbergh, P.H. Vance. 1998. Branch-and-Price: Column Generation for Solving Huge Integer Programs. Operations Research 46 316-329.

Beasley, J.E. 1995. Lagrangean Relaxation. Pages 243-303 of: Reeves, C.R. (ed), Modern Heuristic Techniques for Combinatorial Problems. McGraw-Hill, London.

Dantzig, G.B., P. Wolfe. 1960. Decomposition principles for linear programming. Operations Research 8 01-111.

Desaulniers, G., J. Desrosiers, Y. Dumas, M.M. Solomon, F. Soumis. 1997. Daily aircraft routing and scheduling. Management Sci. 43 841-855.

Desaulniers, G., J. Desrosiers, M.M. Solomon (eds). 2005. Column Generation. Springer, New York.

Eliashberg, J., J.-J. Jonker, M.S. Sawhney, B. Wierenga. 2000. MOVIEMOD: An Implementable Decision Support System for Prerelease Market Evaluation of Motion Pictures Marketing Sci. 19 226243.

Eliashberg, J., S. Swami, C.B. Weinberg, B. Wierenga. 2001. Implementing and evaluating SilverScreener: A marketing management support system for movie exhibitors. Interfaces 31 S108-S127.

Garey , M.R., D.S. Johnson. 1979. Computers and Intractability: a Guide to the Theory of NPCompleteness. Freeman, San Francisco.

Hanssens, D.M., L.J.Parsons, R.L. Schulz. 2003. Market Response Models: Econometric and Time Series Analysis (sec. ed). Kluwer, Boston.

Ho, J. 2005 Marketing Models of Entertainment Products. Unpublished doctoral thesis, University of British Columbia.

Huisman D., R. Freling, A.P.M. Wagelmans. 2005. Multiple-Depot Integrated Vehicle and Crew Scheduling, Transportation Sci. 39 491-502.

Huisman, D., R. Jans, M. Peeters, A.P.M. Wagelmans. 2005. Combining Column Generation and Lagrangian Relaxation. Pages 247-270 of: Desaulniers, G., Desrosiers, J., Solomon, M.M. (eds), Column Generation. Springer, New York.

Krider, R.E., C.B. Weinberg. 1998. Competitive Dynamics and the Introduction of New Products: The Motion Picture Timing Game. Journal of Marketing Research 35 1-15.

Lehmann, D.R., C.B. Weinberg. 2000. Sales through Sequential Distribution Channels: An Application to Movies and Videos. Journal of Marketing 64 18-33.

Löbel, A. 1998. Vehicle scheduling in public transit and Lagrangean pricing. Management Sci. 44 16371649.

Lübbecke, M.E., J. Desrosiers. 2005. Selected Topics in Column Generation. Operations Research 53 1007-1023.

NVB. 2005. Nederlandse Vereniging van Bioscoopexploitanten, Annual Report, Amstelveen, the Netherlands. 
Sandhu, R., D. Klabjan. 2006. Integrated Airline Fleeting and Crew Pairing Decisions. To appear in $O p$ erations Research.

Sawhney, M.S., J. Eliashberg. 1996. A parsimonious model for forecasting gross box-office revenues of motion pictures. Marketing Sci. 15 113-131.

Spann, M. and B. Skiera. 2003. Internet-Based Virtual Stock Markets for Business Forecasting. Management Science 49 1310-1326.

Swami, S., J. Eliashberg, C.B. Weinberg. 1999. SilverScreener: A modeling approach to movie screens management. Marketing Sci. 18 352-372. 


\section{Appendix 1: Definition of Movie Attributes}

$O-U S A_{j} \quad \equiv$ Indicator variable for the event that movie $j$ is made in the U.S.

$O-D U T C H_{j} \quad \equiv$ Indicator variable for the event that movie $j$ is made in the Netherlands

$O-O T H E R_{j} \quad \equiv$ Indicator variable for the event that movie $j$ is made in other countries

$L-E N G L I S H_{j} \quad \equiv$ Indicator variable for the event that movie $j$ is in English

$L-D U T C H_{j} \quad \equiv$ Indicator variable for the event that movie $j$ is in Dutch

$L$-OTHER $\quad \equiv$ Indicator variable for the event that movie $j$ is in other languages

$D-D U T C H_{j} \quad \equiv$ Indicator variable for the event that movie $j$ is dubbed Dutch

$S E Q U E L_{j} \quad \equiv$ Indicator variable for the event that movie $j$ is a sequel

FRANCHISE $_{j} \equiv$ Average worldwide box office gross of all preceding movies in the franchise of movie $j$, if movie $j$ is a sequel.

$B O W 1_{j} \quad \equiv$ Opening week's box office sales in the U.S., if movie $j$ is made in U.S.

$T N W 1_{j} \quad \equiv$ Opening week's theater numbers in the U.S., if movie $j$ is made in U.S.

$B O W 1 W 2_{j} \quad \equiv$ Box office sales percentage change from opening week to the second week in the U.S., if movie $j$ is made in U.S.

$M P A A-G_{j} \quad \equiv$ Indicator variable for the event that movie $j$ is rated $\mathrm{G}$ by MPAA and made in U.S.

$M P A A-P G_{j} \equiv$ Indicator variable for the event that movie $j$ is rated PG and made in U.S.

$M P A A-P G 13_{j} \equiv$ Indicator variable for the event that movie $j$ is rated PG13 and made in U.S.

$M P A A-R_{j} \quad \equiv$ Indicator variable for the event that movie $j$ is rated $\mathrm{R}$ and made in U.S.

$D R A M A_{j} \quad \equiv$ Indicator variable for the event that movie $j$ is classified by Variety.com to be in the drama genre and made in U.S.

$A C T I O N_{j} \quad \equiv$ Indicator variable for the event that movie $j$ is in the action genre and made in U.S.

$C O M E D Y_{j} \equiv$ Indicator variable for the event that movie $j$ is in the comedy genre and made in U.S.

$R C O M E D Y_{j} \equiv$ Indicator variable for the event that movie $j$ is in the romantic comedy genre and made in U.S.

$M U S I C A L_{j} \quad \equiv$ Indicator variable for the event that movie $j$ is in the musical genre and made in U.S.

SUSPENSE $_{j} \quad \equiv$ Indicator variable for the event that movie $j$ is in the suspense genre and made in U.S.

$S_{C I F I} \quad \equiv$ Indicator variable for the event that movie $j$ is in the sci-fi genre and made in U.S.

$H_{O R R O R} \equiv$ Indicator variable for the event that movie $j$ is in the horror genre and made in U.S.

FANTASY $_{j} \equiv$ Indicator variable for the event that movie $j$ is in the fantasy genre and made in U.S.

WESTERN $_{j} \equiv$ Indicator variable for the event that movie $j$ is in the western genre and made in U.S.

$A N I M A T E D_{j} \quad \equiv$ Indicator variable for the event that movie $j$ is in the animated genre and is made in U.S.

$A D V E N T U R E_{j} \equiv$ Indicator variable for the event that movie $j$ is in the adventure genre and made in U.S.

$D O C U_{j} \quad \equiv$ Indicator variable for the event that movie $j$ is in the documentary genre and made in U.S. 
Appendix 2: List of movies for the movie week of March 3-9 2005

\begin{tabular}{|c|c|c|c|c|}
\hline No & Full Name & Abbreviation & Kids? & Duration $^{3}$ \\
\hline 1 & Million Dollar Baby & MDB & No & 147 \\
\hline 2 & Meet The Fockers & MTF & No & 130 \\
\hline 3 & Constantine & $\mathrm{CO} 1$ & No & 136 \\
\hline 4 & Constantine & $\mathrm{CO} 2$ & No & 136 \\
\hline 5 & Melinda And Melinda & MM & No & 114 \\
\hline 6 & The Aviator & AVI & No & 185 \\
\hline 7 & Birth & $\mathrm{BI}$ & No & 115 \\
\hline 8 & Team America & TA & No & 113 \\
\hline 9 & Ray & RAY & No & 167 \\
\hline 10 & Raise Your Voice & RYV & No & 118 \\
\hline 11 & Shall We Dance & SWD & No & 121 \\
\hline 12 & Spongebob & SNL & No & 99 \\
\hline 13 & The Life Aquatic & AQ & No & 133 \\
\hline 14 & Bride And Prejudice & BAP & No & 126 \\
\hline 15 & Hide \& Seek & HS & No & 122 \\
\hline 16 & Vet Hard & $\mathrm{VH}$ & No & 105 \\
\hline 17 & Closer & CLO & No & 119 \\
\hline 18 & Der Untergang & UNT & No & 165 \\
\hline 19 & The Woodsman & WOO & No & 103 \\
\hline 20 & Lepel & LPL & Yes & 104 \\
\hline 21 & Plop \& Kwispel & PK & Yes & 71 \\
\hline 22 & Incredibles & INC & Yes & 130 \\
\hline 23 & Streep Wil Racen & STR & Yes & 104 \\
\hline 24 & Passion Of The Christ ${ }^{1}$ & PAS & No & 126 \\
\hline 25 & Goodbye Lenin ${ }^{1}$ & GL & No & 136 \\
\hline 26 & $\mathrm{Hitch}^{2}$ & HIT & No & 133 \\
\hline \multicolumn{5}{|c|}{ 1) One show, Sunday morning } \\
\hline 2) On & , Saturday night & & & \\
\hline
\end{tabular}




\section{Publications in the Report Series Research ${ }^{*}$ in Management}

\section{ERIM Research Program: "Marketing"}

2007

Marketing Communication Drivers of Adoption Timing of a New E-Service Among Existing Customers

Remco Prins and Peter C. Verhoef

ERS-2007-018-MKT

http://hdl.handle.net/1765/9405

Indirect Network Effects in New Product Growth

Stefan Stremersch, Gerard J. Tellis, Philip Hans Franses and Jeroen L.G. Binken

ERS-2007-019-MKT

http://hdl.handle.net/1765/9406

Demand-Driven Scheduling of Movies in a Multiplex

Jehoshua Eliashberg, Quintus Hegie, Jason Ho, Dennis Huisman, Steven J. Miller, Sanjeev Swami, Charles B. Weinberg and Berend Wierenga

ERS-2007-033-MKT

http://hdl.handle.net/1765/10069

A complete overview of the ERIM Report Series Research in Management:

https://ep.eur.nl/handle/1765/1

ERIM Research Programs:

LIS Business Processes, Logistics and Information Systems

ORG Organizing for Performance

MKT Marketing

F\&A Finance and Accounting

STR Strategy and Entrepreneurship 